\title{
Tác động của lãnh đạo chuyển dạng và lãnh đạo phụng sự đến hoạt động của tổ chức: Vai trò của tổ chức học tập
}

\section{The effect of transformational and servant leadership on organization performance: The role of learning organization}

\author{
Trần Phạm Khánh Toàn ${ }^{1 *}$, Trương Trung Trực ${ }^{2}$ \\ ${ }^{1}$ Trường Đại học Mở Thành phố Hồ Chí Minh, Việt Nam \\ ${ }^{2}$ Trường Đại học Gia Định, Việt Nam \\ "Tác giả liên hệ, Email: toantpk.19ae@ou.edu.vn, khanhtoan014@gmail.com
}

\section{THÔNG TIN}

DOI:10.46223/HCMCOUJS.

Ngày nhận: 05/01/2021

Ngày nhận lại: 05/04/2021

Duyệt đăng: 28/04/2021

\section{Tù khóa:}

doanh nghiệp công nghệ thông tin; hoạt động của tổ chức; lãnh đạo chuyển dạng; lãnh đạo phụng sự; tổ chức học tập; Thành phố Hồ Chí Minh

Keywords:

information technology enterprise; organization performance; transformational leadership; servant leadership; organizational learning, Ho Chi Minh City

\section{TÓM TÁT}

Phong cách lãnh đạo đóng vai trò quan trọng trong việc nâng cao hiệu quả của tổ chức. Mục tiêu của nghiên cứu này nhằm đánh giá tác động của hai phong cách lãnh đạo, bao gồm lãnh đạo chuyển dạng và lãnh đạo phụng sự đến hoạt động của tổ chức thông qua yếu tố tổ chức học tập. Dữ liệu khảo sát được thu thập từ 281 nhân viên đang làm việc tại các doanh nghiệp thuộc lĩnh vực công nghệ thông tin trên địa bàn thành phố Hồ Chí Minh. Mô hình phương trình cấu trúc bình phương nhỏ nhất từng phần (Partial Least Square - Structural Equation Model) được tác giả sử dụng để kiểm định sự phù hợp của mô hình nghiên cứu và các giả thuyết nghiên cứu. Kết quả cho thấy lãnh đạo phụng sự tác động mạnh hơn đến tổ chức học tập so với lãnh đạo chuyển dạng và tổ chức học tập góp phần nâng cao hoạt động của tổ chức.

ABSTRACT
Leadership plays an important role in improving
organizational efficiency. The purpose of this study is to evaluate
the impact of two leadership styles, including transformational
leadership and service leadership, on organization performance
hrough organization learning. Survey data were collected from
281 employees working in information technology enterprises in
Ho Chi Minh City. Partial Least Square - Structural Equation
Model (Partial Least Square) model is used to test the research
model and research hypotheses. The results show that serving
leadership has a stronger impact on the learning organization than
transitional leadership and the learning organization contributes to
improving the organization performance.

\section{Giới thiệu}

Trong hơn một thập kỷ qua, thực tiễn kinh doanh trên thế giới đã đặt ra những vấn đề đầy thách thức cho các nhà quản lý trong đó có việc sử dụng phong cách lãnh đạo phù hợp với tình hình mới. Vai trò của người lãnh đạo vô cùng quan trọng trong các tổ chức, là yếu tố có tính quyết 
định đến sự thành công, khả năng tồn tại phát triển của một tổ chức, một doanh nghiệp (Olesia, Namusonge, \& Iravo, 2014; Staats, 2015). Một người lãnh đạo với phong cách lãnh đạo phù hợp sẽ thúc đẩy cho tổ chức phát triển, sẽ là điều kiện tốt nhất cho những người lao động trong tổ chức đó ngày càng phát triển hơn về trình độ chuyên môn, kỹ năng, ngày càng hoàn thiện bản thân, tổ chức ngày càng phát triển và ngược lại.

Lãnh đạo chuyển dạng (transformational leadership) ảnh hưởng đến thái độ, hành vi của nhân viên và các đặc điểm của tổ chức (Garcia-Morales, Lloens-Montes, \& Verdu-Jover, 2008) còn lãnh đạo phụng sự (servant leadership) được hiểu là việc ưu tiên phát triển nhân viên nhằm giúp họ phát huy năng lực (Ehrhart, 2004). Hiện nay, trên thế giới đã có nhiều nghiên cứu về tác động của lãnh đạo chuyển dạng đến hoạt động của tổ chức (Malcalm \& Tametey, 2017; Voon, Lo, Ngui, \& Ayob, 2011) cũng như là lãnh đạo phụng sự đến hoạt động của tổ chức (Lapointe \& Vandernberghe, 2018; Liu, 2019), tuy nhiên, chưa có nghiên cứu cụ thể nào so sánh tác động của hai phong cách đến hoạt động của tổ chức thông qua yếu tố tổ chức học tập (organizational learning). Đặc biệt khi tổ chức học tập là một yếu tố quan trọng, quyết định sự phát triển bền vững của tổ chức (López, Peón, \& Ordás, 2005; Prugsamatz, 2010) bởi vì học tập tốt hơn và nhanh hơn sẽ giúp các tổ chức thích ứng tốt hơn trong môi trường thay đổi nhanh chóng (Baldwin, 2016; Örtenblad, 2018). Mặc dù có nhiều nghiên cứu cho thấy sự quan hệ tích cực giữa khả năng lãnh đạo và hoạt động học tập trong đơn vị nhưng rất ít nghiên cứu về phong cách lãnh đạo và sự học tập của tổ chức (Gentle \& Clifton, 2017; Schiena, Letens, Aken, \& Farris, 2013). Một tổ chức có tính chất học tập là một tổ chức tạo điều kiện cho việc học tập và thúc đẩy kết quả học tập ở nhiều cấp độ (cá nhân, nhóm, đội, và tổ chức) nhằm nâng cao hoạt động của tổ chức (Garvin, Edmondson, \& Gino, 2008). Do đó, việc đi sâu nghiên cứu, phân tích tác động của phong cách lãnh đạo chuyển dạng và phụng sự thông qua yếu tố tổ chức học tập là hết sức cần thiết, góp phần tạo điều kiện cho các nhà quản lý trong các doanh nghiệp đạt được mục tiêu phát triển bền vững.

Trong những năm vừa qua, khu vực doanh nghiệp công nghệ thông tin phát triển rất mạnh mẽ ở Việt Nam nói chung và ở Thành phố Hồ Chí Minh nói riêng. Đa số các doanh nghiệp công nghệ thông tin đều áp dụng các phương thức quản lý tiên tiến, hiệu quả, trong đó nhấn mạnh vai trò của người lãnh đạo, quản lý. Hơn nữa, trong lĩnh vực công nghệ thông tin đòi hỏi các nhà quản lý phải tạo điều kiện thuận lợi cho các nhân viên liên tục học tập, chia sẻ tri thức, không ngừng đổi mới sáng tạo. Do đó, việc hình thành và duy trì tổ chức học tập là một trong những yếu tố góp phần nâng cao tính cạnh tranh, hiệu quả hoạt động trong dài hạn. Đa số các nghiên cứu trước đây về tác động của phong cách lãnh đạo đến hoạt động của tổ chức thông qua yếu tố tổ chức học tập đều được thực hiện ở khu vực Châu Âu và Hoa Kỳ (Garcia-Morales, Jimenez-Barrionuevo, \& Gutierrez-Gutierrez, 2012; Johnson, 2002) và dường như chưa thu hút được sự quan tâm nghiên cứu ở Việt Nam, đặc biệt là trong lĩnh vực công nghệ thông tin. Việc đi sâu nghiên cứu mối quan hệ này sẽ bổ sung cho khoảng trống nghiên cứu đó. Kết quả nghiên cứu sẽ giúp cho các nhà lãnh đạo, quản lý ở các doanh nghiệp công nghệ thông tin nâng cao hiệu quả hoạt động của tổ chức bằng cách sử dụng các phong cách lãnh đạo thích hợp và xây dựng, nuôi dưỡng tổ chức học tập.

Phần tiếp theo của nghiên cứu sẽ trình bày cơ sở lý thuyết và mô hình nghiên cứu và các giả thuyết nghiên cứu đề xuất. Tại phần 3 sẽ giới thiệu thiết kê nghiên cứu, phần 4 trình bày kết quả nghiên cứu và cuối cùng sẽ là kết luận và hàm ý chính sách.

\section{Cơ sở lý thuyết và mô hình nghiên cứu}

\subsection{Các khái niệm}

\subsubsection{Lãnh đạo chuyển dạng}

Lãnh đạo chuyển dạng đã thu hút được sự quan tâm và trở thành sự lựa chọn cho việc 
nghiên cứu và ứng dụng của các lý thuyết lãnh đạo. Burns (1978) đã giới thiệu thuật ngữ lãnh đạo chuyển dạng nhằm mô tả mối quan hệ tối ưu giữa các nhà lãnh đạo chính trị và những người nhân viên. Bass (1985) đã phát triển khái niệm này và giới thiệu vào lý thuyết phong cách lãnh đạo. Theo đó, lãnh đạo chuyển dạng là truyền cảm hứng cho nhân viên, cam kết một tầm nhìn chung và mục tiêu cho tổ chức, thách thức, thúc đẩy những nhân viên của mình để giải quyết vấn đề sáng tạo, đạt được các mục tiêu của tổ chức và do đó nâng cao hiệu suất công việc tổng thể (Bass, 1985; Bass \& Riggo, 2006). Podsakoff, MacKenzie, và Bommer (1996) cho rằng các nhà quản lý có phong cách lãnh đạo chuyển dạng cần phải vượt lên trên tư lợi vì lợi ích của tổ chức. Điều này hàm ý rằng nhân viên được thúc đẩy bởi các nhà lãnh đạo chuyển dạng, những người hy sinh lợi ích cá nhân của họ cho sự phát triển của tổ chức (Tepper et al., 2017).

Các nghiên cứu đều nhấn mạnh đến khía cạnh tầm nhìn dài hạn là đặc điểm chính của các nhà lãnh đạo chuyển dạng. Tầm nhìn của họ tạo ra các điều kiện nhằm nâng cao sự gắn kết với công việc, hiệu suất công việc, thúc đẩy học tập và chia sẻ tri thức và phát triển bền vững trong các môi trường văn hóa (Jyoti \& Dev, 2015; Geier, 2016; Triana, Richard, \& Yucel, 2017). Bên cạnh đó, lãnh đạo chuyển dạng giúp nhân viên có một môi trường làm việc thân thiện, hỗ trợ, giảm căng thẳng và xung đột giữa công việc và gia đình (Arnold, 2017). Về khía cạnh học tập, Lam (2002) nhận thấy lãnh đạo chuyển dạng có ảnh hưởng tích cực đến quá trình và kết quả học tập của tổ chức trong môi trường đa văn hóa, đa quốc gia.

\subsubsection{Lãnh đạo phụng sụ}

Gần đây, lãnh đạo phụng sự trở thành một khái niệm chủ đạo trong nghiên cứu về phong cách lãnh đạo (Dierendonck, 2011; Ehrhart, 2004; Hoch, Bommer, Dulebohn, \& Wu, 2018; Sendjaya, Sarros, \& Santora, 2008). Quá trình phát triển của thuật ngữ lãnh đạo phụng sự đã trải dài hơn một thập kỷ (Stone, Russell, \& Patterson, 2004). Khái niệm lãnh đạo phụng sự là một khái niệm khá phức tạp (Dierendonck \& Nuijten, 2011). Lãnh đạo phụng sự được xem như là một triết lý lãnh đạo giải quyết các mối quan tâm về đạo đức (Carter \& Baghurst, 2014), nó có mối liên hệ với lãnh đạo theo đạo đức, đức hạnh, liêm chính (Lanctot \& Irving 2010; Parris \& Peachey, 2013).

Greenleaf (1977) cho rằng lãnh đạo phụng sự là đặt nhu cầu của người lao động trước nhu cầu riêng của họ và tập trung nỗ lực vào việc giúp người lao động phát triển đạt được tiềm năng tối đa và đạt được sự thành công của tổ chức. Tuy đồng thuận về mặt cơ bản là các nhà quản lý theo phong cách lãnh đạo phụng sự đặt nhân viên của mình lên hàng đầu và giúp họ phát huy hết tiềm năng của mình (Northouse, 2018; Sendjaya et al., 2008; Spears, 2010), từ đó thúc đẩy hiệu quả hoạt động của cơ quan, tổ chức (Lapointe \& Vandernberghe, 2018; Liu, 2019).

Theo quan điểm của Russell và Stone (2002), Dierendonck (2011), và Parris và Peachey (2013), các nhà lãnh đạo phụng sự tìm kiếm một nhu cầu hoạt động cao hơn, và động lực chính trong hoạt động của họ là mong muốn phục vụ những nhân viên đi theo họ. Cả hai chiều của lãnh đạo phụng sự là "phục vụ" và "lãnh đạo" đều rất quan trọng cho sự thịnh vượng của một tổ chức (Ragnarsson, Kristjánsdóttir, \& Gunnarsdottir, 2018). Đây là triết lý chính của lãnh đạo phụng sự.

Những đặc điểm của lãnh đạo phụng sự dường như mâu thuẫn với tiền đề của hoạt động học hỏi của tổ chức. Một tổ chức học tập phải gắn với mục tiêu tập thể rõ ràng, cụ thể thay vì mục tiêu cá nhân phân tán (Senge, 1990). Lãnh đạo phụng sự có tác động mạnh mẽ đến việc chia sẻ kiến thức và học tập trong tổ chức (Choudhary, Akhtar, \& Zaheer, 2013; Song, Park, \& Kang, 2015). Tuy nhiên, Sendjaya (2015) cho rằng phương pháp lãnh đạo phụng sự không hướng đến hiệu quả vì nó không hy sinh nhân viên để đạt được lợi nhuận và tăng trưởng.

\subsubsection{Lãnh đạo chuyển dạng và lãnh đạo phụng sụ}


Mặc dù lãnh đạo phụng sự chia sẻ một số đặc điểm tương đồng với lãnh đạo chuyển dạng (Dierendonck, Stam, Boersma, Windt, \& Alkema, 2014) nhưng có những đặc điểm riêng biệt, không sử dụng thay thế cho nhau (Ehrhart, 2004; Hoch et al., 2018). Lãnh đạo chuyển dạng là cấp có thẩm quyền cao và cung cấp một chương trình nghị sự rõ ràng và hấp dẫn và truyền cảm hứng cho nhân viên để thực hiện chương trình, kế hoạch đó (Hackman, 2002). Tuy nhiên, không giống như một nhà lãnh đạo chuyển dạng - luôn tập trung vào mục tiêu tổ chức, một lãnh đạo phụng sự dành sự quan tâm đối với những nhân viên của mình, sự khiêm tốn, tính chính trực, đồng cảm (Dierendonck, 2011).

\subsubsection{Tổ chức học tập}

Tổ chức học tập là một yếu tố quan trọng để nâng cao hiệu quả hoạt động của tổ chức, đơn vị trong dài hạn (Örtenblad, 2018; Peltokorpi \& Niemi, 2018; Veelen \& Ufkes, 2017). Tổ chức học tập được định nghĩa chung nhất là một hệ thống mở nhằm nuôi dưỡng, phát triển văn hóa học tập của tổ chức, tạo điều kiện cho các hoạt động học tập, phổ biến kết quả học tập và sử dụng học tập để nâng cao hiệu suất hoạt động (Xie, 2020). Trong một tổ chức có đặc tính học tập, nhân viên sẽ liên tục học tập, sáng tạo, tiếp thu và chuyển giao kiến thức - giúp công ty của họ thích ứng với những điều khó đoán định nhanh hơn so với các công ty cạnh tranh (Garvin et al., 2008). Senge (1990) nhấn mạnh tầm quan trọng của một nền văn hóa khuyến khích học tập, tư duy sáng tạo trong bất kỳ tổ chức nào. Tổ chức học tập luôn thúc đẩy sự cải tiến liên tục trong cấu trúc tổ chức và có lợi cho sự phát triển và hiệu suất của nhân viên (Garcia-Morales et al., 2008).

\subsubsection{Hoạt động của tổ chức}

Hiệu quả hoạt động là vấn đề quan trọng liên quan đến kết quả đầu ra của tổ chức và sự thành công của tổ chức (Campbell, 1990). Cho và Dansereau (2010) đề cập đến hiệu quả hoạt động của doanh nghiệp khi so sánh với mục tiêu, kế hoạch. Ngoài ra, Tomal và Jones (2015) định nghĩa hiệu quả hoạt động của tổ chức là kết quả thực tế hoặc đầu ra của tổ chức. Có thể xem xét hiệu quả hoạt động của tổ chức dưới nhiều góc độ khác nhau như: giảm chi phí, tăng lợi nhuận, doanh số bán hàng, sự hài lòng của khách hàng, ... (Rhodes, Lok, Hung, \& Fang, 2008).

\subsection{Các giả thuyết nghiên cứu và mô hình nghiên cứu}

\subsubsection{Mối quan hệ giũa lãnh đạo chuyển dạng và lãnh đạo phụng sụ và tổ chức học tập}

Về khía cạnh xây dựng tổ chức học tập, cả hai phương pháp lãnh đạo đều có tác động tích cực (Chang \& Lee, 2007; Singh, 2008; Tan, Smyrnios, \& Xiong, 2014). Lãnh đạo chuyển dạng là yếu tố quan trọng trong việc thúc đẩy quá trình học tập trong tổ chức (Senge, 1990). Lãnh đạo chuyển dạng tác động trực tiếp đến sự thay đổi, chuyển đổi của đơn vị, doanh nghiệp thông qua sự chuyển đổi cá nhân. Lãnh đạo chuyển dạng giúp nhân viên đạt được mục tiêu tập thể (Bass, 2000; Stone et al., 2004). Lãnh đạo chuyển dạng trao quyền cho mọi người học tập, phát triển sáng tạo đặc biệt là trong môi trường cạnh tranh như hiện nay (Dimmock \& Walker, 2005; Parolini, Pateerson, \& Winston, 2009).

So với lãnh đạo chuyển dạng thì các nhà quản lý theo phương pháp lãnh đạo phụng sự sẽ khuyến khích nhân viên học tập và từ đó phát triển, thúc đẩy tạo nên văn hóa học tập giữa các nhân viên (Bass, 2000). Môi trường học tập của tổ chức được hỗ trợ nâng cao bởi phong cách lãnh đạo phụng sự (Crippen, 2005; McClellan, 2007). Một khi nhà quản lý theo dõi nhu cầu phát triển của nhân viên sẽ tổ chức đào tạo, bồi dưỡng cho họ, từ đó làm tăng khả năng học tập của tổ chức (Xie, 2019).

Như đã trình bày ở trên, giữa lãnh đạo chuyển dạng và lãnh đạo phụng sự có nhiều điểm tương đồng nhưng vẫn tồn tại những khác biệt cơ bản (Hoch et al., 2018). Qua khảo sát các nghiên cứu trước, lãnh đạo chuyển dạng và lãnh đạo phụng sự có thể tác động đến sự học tập của tổ chức, 
do vậy hai giả thuyết được đặt ra:

H1: Lãnh đạo chuyển dạng có tác động tích cưc đến sụ học tập của tổ chức

H2: Lãnh đạo phụng sụ có tác động tích cực đến sụ học tập của tổ chức

\subsubsection{Mối quan hệ giũa tổ chức học tập và hoạt động của tổ chức}

Tổ chức học tập thúc đẩy hoạt động của tổ chức đã được nhiều nghiên cứu minh chứng (Akhtar, Arif, Rubi, \& Naveed, 2012; Ho, 2011; Khandekar \& Sharma, 2006). Hơn nữa, sự học tập của tổ chức có tác động tích cực đến việc tích lũy, chia sẻ và phát triển tri thức, từ đó tác động tích cực đến hiệu quả hoạt động của tổ chức, đơn vị. Điều này là có nguyên nhân là do tổ chức đó có một môi trường học tập liên tục và hài hòa (Akhtar et al., 2012) khuyến khích các cá nhân cam kết nhiều hơn trong việc đạt được các mục tiêu của công ty, khuyến khích chia sẻ thông tin và học hỏi. Tuy nhiên, cũng có nhiều nghiên cứu cho thấy tác động tổ chức học tập đối với hoạt động kinh doanh khác nhau, nhưng nhìn chung thì có tác động tích cực (Garcia-Morales et al., 2008; Morgan \& Turnell, 2003). Do vậy, giả thuyết được đặt ra:

H3: Sụ học tập của tổ chức tác động tích cưc đến hoạt động của tổ chức

\section{Bảng 1}

Tóm tắt các nghiên cứu trước có liên quan

\section{Mối quan hệ giữa 2 yếu tố Các nghiên cứu trước}

Lãnh đạo chuyển dạng $\rightarrow$ Senge (1990), Sarros, Tanewski, Winter, Santora, và Densten Sự học tập của tổ chức ～(2002), Bono và Judge (2003), Garcia-Morales và cộng sự (2008)

Lãnh đạo phụng sự $\rightarrow$ Bass (2000), Crippen (2005), McClellan (2007), Xie (2019), Chang Sự học tập của tổ chức và Lee (2007), Singh (2008); Tan và cộng sự (2014)

Sự học tập của tổ chức $\rightarrow$ Morgan và Turnell (2003), Garcia-Morales và cộng sự (2008) Hoạt động của tổ chức

Nguồn: Tác giả tổng hợp

\subsubsection{Mô hình nghiên cúu đề xuất}

Trên cơ sở lược khảo các nghiên cứu trước có liên quan, mô hình nghiên cứu đề xuất như Hình 1. Theo đó, nghiên cứu sẽ kiểm tra tác động của phong cách lãnh đạo chuyển dạng đến sự học tập của tổ chức; tác động của phong cách lãnh đạo phụng sự đến sự học tập của tổ chức và tác động của sự học tập của tổ chức đến hiệu quả hoạt động của tổ chức.

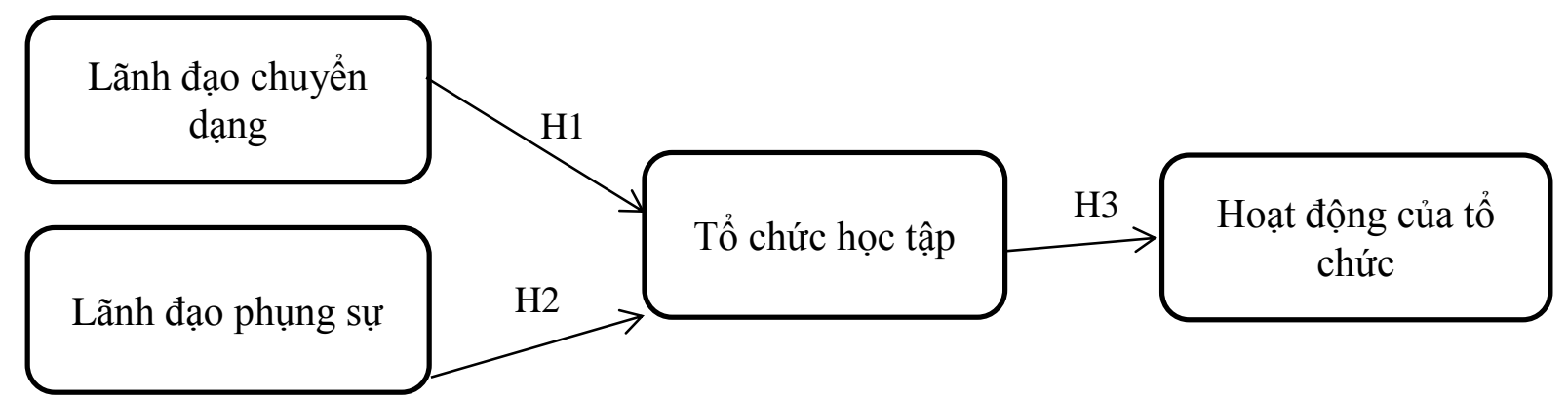

Hình 1. Mô hình nghiên cứu 


\section{Thiết kế nghiên cứu}

\subsection{Phưong pháp nghiên cúu}

Trong quá trình thực hiện, nghiên cứu kết hợp phương pháp nghiên cứu định lượng và nghiên cứu định tính. Phương pháp định tính bao gồm các hoạt động lấy ý kiến chuyên gia được sử dụng trong xây dựng thang đo phong cách lãnh đạo phụng sự, lãnh đạo chuyển dạng Nghiên cứu sử dụng phương pháp định lượng thông qua các kỹ thuật thống kê mô tả, kiểm định độ tin cậy thang đo, phân tích nhân tố khám phá, phân tích mô hình phương trình cấu trúc.

\subsection{Thang do}

Qua phần khảo sát định tính dưới hình thức lấy ý kiến chuyên gia (nhà nghiên cứu quản trị nhân sự, nhà quản lý doanh nghiệp, và các nhân viên có kinh nghiệm công tác) nội dung thang đo các khái niệm là phù hợp với bối cảnh tại Việt Nam. Thang đo lãnh đạo chuyển dạng được dựa trên nghiên cứu của Garcia-Morales và cộng sự (2008) gồm 05 biến quan sát (được ký hiệu là TL), thang đo phong cách lãnh đạo phụng sự được dựa trên nghiên cứu của Jacobs (2006) gồm 05 biến quan sát (được ký hiệu là SL). Tuy nhiên, Jacobs (2006) sử dụng thang đo Likert bảy điểm, do vậy nghiên cứu chuyển đổi thành thang đo năm điểm nhằm thuận tiện và thống nhất trong bảng câu hỏi khảo sát. Thang đo sự học tập của tổ chức bao gồm 05 biến quan sát (được ký hiệu là OL) được trích từ nghiên cứu của Garcia-Morales và cộng sự (2008). Một trong số 05 quan sát là một câu hỏi mở đã được điều chỉnh thành thang đo Likert năm điểm. Cuối cùng là thang đo hiệu quả hoạt động của tổ chức được trích từ nghiên cứu của Garcia-Morales và cộng sự (2008) bao gồm 05 biến quan sát (được ký hiệu là $\mathrm{OP}$ ).

\subsection{Dũu liệu nghiên cúu}

Nghiên cứu sử dụng phương pháp chọn mẫu thuận tiện để thực hiện, đối tượng thu thập thông tin phục vụ cho nghiên cứu là các nhân viên đang làm việc tại các doanh nghiệp ngành công nghệ thông tin đang hoạt động tại Thành phố Hồ Chí Minh trong thời gian từ tháng 01 đến tháng 02/2021. Đầu tiên, nhóm tác giả liên hệ Ban Quản lý Khu Công nghệ cao Thành phố Hồ Chí Minh để xin phép được khảo sát. Được sự đồng ý và giới thiệu của lãnh đạo Ban, nhóm tác giả tiến hành xin lịch hẹn để tiến hành trao đổi triển khai thực hiện khảo sát. Kết quả có 12 doanh nghiệp đồng ý tham gia khảo sát.

Trước khi thực hiện khảo sát tại từng công ty, nhóm tác giả đã trình bày, giải thích cho đại diện nhóm nhân viên về mô hình nghiên cứu, các phát biểu trong bảng hỏi cũng như cách thức trả lời, đồng thời nhờ họ về hướng dẫn lại cho các nhân viên khác và hẹn sau một tuần sẽ đến nhận lại phiếu khảo sát.

Theo nguyên tắc, cỡ mẫu nghiên cứu càng lớn là càng tốt và theo Hair, Hult, Ringle, và Sarstedt (2014) thì cỡ mẫu tối thiểu cho mô hình SEM sẽ bằng ít nhất 05 lần số biến quan sát. Mô hình nghiên cứu đề xuất có 25 biến quan sát, do đó cỡ mẫu tối thiểu phải là 125 . Tác giả phát ra 350 phiếu khảo sát, kết quả thu về có 304 phản hồi (đạt tỷ lệ 86,86\%). Sau khi làm sạch dữ liệu, dữ liệu chính thức là 281 và được xử lý bằng phần mềm SPSS và Smart - PLS 3.2.7.

\section{Kết quả nghiên cứu}

\subsection{Thống kê mô tả}

Trong 281 đáp viên, số lượng người có giới tính Nam là 167 người, chiếm tỷ lệ $59.43 \%$ và giới tính Nữ là 114 người, chiếm tỷ lệ $40.57 \%$. Về đặc điểm độ tuổi, kết quả cho thấy đối tượng được khảo sát ở nhóm tuổi từ 22 đến 30 tuổi chiếm tỷ lệ cao nhất với 127 người, tỷ lệ $45.2 \%$; kế tiếp là những người thuộc nhóm tuổi từ 30 đến 35 tuổi với 102 người và cuối cùng có 52 người 
trên 35 tuổi. Đại đa số người được khảo sát có trình độ Đại học trở lên, với 195 người có trình độ đại học, chiếm 69.4\% và 48 người có trình độ sau đại học, chiếm 17.08\%. Ngoài ra, có 38 người có trình độ Trung cấp, Cao đẳng chiếm tỷ lệ $13.52 \%$.

\subsection{Phân tích độ tin cậy và tính giá trị của mô hình}

Kết quả phân tích lần đầu cho thấy giá trị Cronbach's Alpha của thang đo OL5 nhỏ hơn 0.7 , do đó loại thang đo OL5 và tiếp tục phân tích. Kết quả phân tích lần 02 được trình bày ở Bảng 2 cho thấy giá trị Cronbach's Alpha và hệ số outer loading đều lớn hơn 0.7 nên độ tin cậy của thang đo là tốt, (Hair et al., 2014). Do vậy, tất cả các biến quan sát đều được chấp nhận.

\section{Bảng 2}

Kết quả phân tích Cronbach's Alpha

\begin{tabular}{llccc}
\hline \multicolumn{1}{c}{ Variable } & & Loadings & Mean & Std.Dev \\
\hline & TL1 & 0.762 & 4.196 & 0.660 \\
Lãnh đạo chuyển dạng (TL) & TL2 & 0.777 & 4.157 & 0.673 \\
Cronbach's Alpha =0.834 & TL3 & 0.793 & 4.181 & 0.710 \\
& TL4 & 0.779 & 4.249 & 0.639 \\
& TL5 & 0.763 & 4.214 & 0.678 \\
\hline & SL1 & 0.756 & 4.206 & 0.755 \\
Lãnh đạo phụng sự (SL) & SL2 & 0.820 & 4.228 & 0.551 \\
Cronbach's Alpha $=0.832$ & SL3 & 0.745 & 4.210 & 0.756 \\
& SL4 & 0.728 & 4.217 & 0.749 \\
\hline Tổ chức học tập (OL) & SL5 & 0.798 & 4.238 & 0.757 \\
Cronbach's Alpha $=0.818$ & OL1 & 0.811 & 4.174 & 0.598 \\
& OL2 & 0.767 & 4.164 & 0.627 \\
& OL3 & 0.802 & 4.171 & 0.642 \\
Hiệu quả hoạt động của tổ chức & OP2 & 0.786 & 4.146 & 0.622 \\
\hline Cronbach's Alpha $=0.844$ & OP3 & 0.786 & 4.142 & 0.656 \\
\hline & OP4 & 0.773 & 4.135 & 0.634 \\
\hline & OP5 & 0.808 & 4.185 & 0.638 \\
\hline & & 0.836 & 4.210 &
\end{tabular}

Nguồn: Kết quả phân tích

Theo kết quả trong Bảng 3 cho thấy độ tin cậy tổng hợp (Composite Reliability - CR) của tất cả các biến đều lớn hơn 0.7 , thấp nhất là 0.879 , và tổng phương sai trích trung bình (Average Variance Extracted - AVE) đều lớn hơn 0.5 , thấp nhất là 0.593 . Từ đó, kết luận mô hình đạt yêu cầu về sự hội tụ. Ngoài ra, bình phương hệ số tương quan lớn nhất (MSV) đều nhỏ hơn phương sai trích $(\mathrm{AVE})$ và giá trị lớn nhất của $\mathrm{HTMT}$ là 0.719 (giữa cặp $\mathrm{OL}$ và $\mathrm{OP}$ ) nhỏ hơn 0.85 . Từ kết quả trên, tất các nhân tố đều đảm bảo độ phân biệt. 


\section{Bảng 3}

Kết quả phân tích $\mathrm{CR}$ và $\mathrm{AVE}$

\begin{tabular}{|c|c|c|c|c|c|c|}
\hline & OL & OP & SL & TL & CR & AVE \\
\hline OL & $\mathbf{0 . 8 0 4}$ & & & & 0.880 & 0.646 \\
\hline OP & 0.600 & $\mathbf{0 . 7 8 5}$ & & & 0.889 & 0.616 \\
\hline SL & 0.648 & 0.605 & $\mathbf{0 . 7 7 0}$ & & 0.879 & 0.593 \\
\hline TL & 0.497 & 0.510 & 0.511 & $\mathbf{0 . 7 7 5}$ & 0.882 & 0.600 \\
\hline
\end{tabular}

Nguồn: Kết quả phân tích

\subsection{Kiểm định mô hình cấu trúc và các giả thuyết}

Kết quả phân tích cho thấy giá trị thống kê Chi - bình phương là 488.847 với $\mathrm{p}$ - value $=$ 0.000 nhỏ hơn 0.005 . Ngoài ra, giá trị SRMR là 0.079 nhỏ hơn 0.1 thì dữ liệu phù hợp với thực tế.

Từ Bảng 4 có thể thấy Kiểm định hiện tượng đa cộng tuyến đều cho giá trị $\mathrm{VIF}<3$, với giá trị lớn nhất là 1.452 nên mô hình không vi phạm hiện tượng đa cộng tuyến. Các biến OL và OP được giải thích bởi $45.3 \%$ và $35.7 \%$ sự thay đổi của các biến độc lập. Ngoài ra, tất cả các chỉ số $\mathrm{Q} 2$ đều lớn hơn $0(\mathrm{Q} 2 \mathrm{OL}=0.283$ và $\mathrm{Q} 2 \mathrm{OP}=0.214)$.

\section{Bảng 4}

Kết quả hệ số VIF, $\mathrm{R}^{2}$ hiệu chỉnh, $\mathrm{f}^{2}, \mathrm{Q}^{2}$

\begin{tabular}{|c|c|c|c|c|c|c|c|}
\hline & \multicolumn{2}{|c|}{ VIF } & \multirow{2}{*}{$\mathbf{R}^{2}$} & \multirow{2}{*}{$\begin{array}{l}\mathbf{R}^{2} \text { hiệu } \\
\text { chỉnh }\end{array}$} & \multicolumn{2}{|c|}{$\mathbf{f}^{2}$} & \multirow[t]{2}{*}{$\mathbf{Q}^{2}$} \\
\hline & OL & $\mathrm{OP}$ & & & OL & $\mathrm{OP}$ & \\
\hline $\mathrm{OL}$ & & 1.00 & 0.457 & 0.453 & & 0.562 & 0.283 \\
\hline $\mathrm{OP}$ & 1.354 & & 0.360 & 0.357 & & & 0.214 \\
\hline $\mathrm{SL}$ & 1.452 & & & & 0.386 & & \\
\hline $\mathrm{TL}$ & & & & & 0.069 & & \\
\hline
\end{tabular}

Nguồn: Kết quả phân tích

Kết quả kiểm định các giả thuyết nghiên cứu được trình bày trong Bảng 5. Kết quả phân tích đường dẫn cho thấy tất cả các giả thuyểt đều được chấp nhận.

\section{Bảng 5}

Kết quả kiểm định giả thuyết nghiên cứu

\begin{tabular}{|l|c|c|c|c|}
\hline \multicolumn{1}{|c|}{ Giả thuyết } & Kỳ vọng & Hệ số chuẩn hóa & P - value & Kết quả \\
\hline $\begin{array}{l}\text { Lãnh đạo chuyển dạng có tác } \\
\text { dộng tích cực đến sự học tập của } \\
\text { tổ chức }\end{array}$ & + & 0.225 & $\begin{array}{c}* * * \\
(\mathrm{P}<0.001)\end{array}$ & Chấp nhận \\
\hline $\begin{array}{l}\text { Lãnh đạo phụng sự có tác động } \\
\text { tích cực đển sự học tập của tồ } \\
\text { chức }\end{array}$ & + & 0.533 & $\begin{array}{c}* * * \\
(\mathrm{P}<0.001)\end{array}$ & Chấp nhận \\
\hline $\begin{array}{l}\text { Sự học tập của tổ chức tác động } \\
\text { tích cực đến hiệu quả hoạt động } \\
\text { của tồ chức }\end{array}$ & + & 0.600 & $\begin{array}{c}* * * \\
(\mathrm{P}<0.001)\end{array}$ & Chấp nhận \\
\hline
\end{tabular}

Nguồn: Kết quả phân tích 


\subsection{Thảo luận kết quả nghiên cứu}

Nghiên cứu này so sánh tác động của lãnh đạo chuyển dạng và lãnh đạo phụng sự đến hoạt động của tổ chức thông qua yếu tố sự học tập của tổ chức của các doanh nghiệp hoạt động trong lĩnh vực công nghệ thông tin trên địa bàn Thành phố Hồ Chí Minh. Nghiên cứu này đã đóng góp vào lý thuyết về phong cách lãnh đạo và lý thuyết về tổ chức học tập theo một số hướng sau:

Một là, lý thuyết tổ chức học tập đã được sử dụng để kết nối hai phong cách lãnh đạo. Các nhà nghiên cứu cho rằng khái niệm tổ chức học tập rất đa dạng và phong phú và cần phải có thêm nhiều nghiên cứu thực nghiệm để minh chứng lý thuyết tổ chức học tập. Kết quả nghiên cứu cho thấy cả hai phong cách lãnh đạo chuyển dạng và lãnh đạo phụng sự đều có tác động tích cực đến tổ chức học tập của các doanh nghiệp. Kết quả này hoàn toàn phù hợp với nghiên cứu Choudhary và cộng sự (2013), Chang và Lee (2007), Singh (2008), Tan và cộng sự (2014). Tuy nhiên, kết quả nghiên cứu này không ủng hộ nghiên cứu của Xie (2020) khi cho rằng lãnh đạo phụng sự không tác động đến sự học tập của tổ chức.

Mối quan hệ giữa lãnh đạo và tổ chức học tập đã được các nhà nghiên cứu quan tâm thảo luận và xác nhận tầm quan trọng của sự lãnh đạo trong việc thiết kế và duy trì một tổ chức học tập (Bass, 2000). Tuy nhiên, có rất ít các nghiên cứu thực nghiệm nhằm kết nối, phân tích hai khái niệm này. Nghiên cứu này đã góp phần bổ sung vào lý thuyết lãnh đạo và tổ chức học tập.

Hai là, kết quả nghiên cứu cho thấy mức độ tác động của phong cách lãnh đạo phụng đến sự học tập của tổ chức mạnh hơn lãnh đạo chuyển dạng. Kết quả này trái ngược với kết quả nghiên cứu của Choudhary và cộng sự (2013). Phát hiện này có thể được giải thích là do phong cách lãnh đạo phụng sự có ảnh hưởng mạnh hơn trong các môi trường có khoảng cách quyền lực hẹp và mọi người bình đẳng hơn. Trong các doanh nghiệp công nghệ thông tin, do áp dụng các phương pháp quản lý hiện đại, hiệu quả nên khoảng cách về quyền lực giữa người lãnh đạo và nhân viên thu hẹp và nhân viên được các nhà quản lý trao cơ hội để thể hiện năng lực, nói lên những suy nghĩ của bản thân. Hơn nữa, tác động này còn có thể được giải thích là do khi xây dựng một tổ chức học tập, người lãnh đạo bắt buộc phải khuyến khích nhân viên tự nghiên cứu, học tập, trao đổi và từ đó phát triển, thúc đẩy tạo nên văn hóa học tập giữa các nhân viên (Bass, 2000).

Ba là, tác động cùng chiều giữa tổ chức học tập và hoạt động của tổ chức được nghiên cứu xác nhận. Kết quả này hoàn toàn phù hợp với các nghiên cứu trước của Akhtar và cộng sự (2012), Morgan và Turnell (2003), Garcia-Morales và cộng sự (2008). Một tổ chức có một môi trường học tập liên tục và hài hòa sẽ khuyến khích các cá nhân cam kết nhiều hơn, nỗ lực nhiều hơn trong việc thực hiện công việc và đạt được các mục tiêu của công ty.

\section{Kết luận và hàm ý chính sách}

Nghiên cứu này phân tích tác động của hai phong cách lãnh đạo là lãnh đạo chuyển dạng và lãnh đạo phụng sự đến hoạt động của tổ chức thông qua yếu tố tổ chức học tập. Kết quả cho thấy các mối quan hệ đều là tích cực. Cụ thể, lãnhih đạo chuyển dạng và lãnh đạo phụng sự đều tác động cùng chiều đến tổ chức học tập, đồng thời tổ chức học tập tác động của chiều đến hoạt động của tổ chức. Ngoài ra, kết quả nghiên cứu cho thấy mức độ tác động của lãnh đạo phụng sự mạnh hơn so với lãnh đạo chuyển dạng đối với tổ chức học tập.

Nghiên cứu cung cấp một số hàm ý quản trị cho các nhà lãnh đạo, quản lý của doanh nghiệp công nghệ thông tin. Cụ thể, họ nên được khuyến khích thực hành khả năng lãnh đạo phụng sự, người quản lý nên lãnh đạo theo đạo đức, liêm chính và đặt nhân viên của mình lên hàng đầu và giúp họ phát huy hết tiềm năng, năng lực. Cần phát huy các trí tuệ cảm xúc, hiểu cảm giác của nhân viên để từ đó nhẹ nhàng hơn trong trao đổi, góp ý; cởi mở hơn nữa trong việc lắng nghe quan 
điểm của người khác. Lãnh đạo phải biết gần gũi, chia sẻ với nhân viên, thường xuyên gặp gỡ, thăm hỏi, động viên nhân viên. Điều này tạo nên sự khích lệ, động viên, cho cấp dưới trong thực thi nhiệm vụ. Đồng thời, lãnh đạo cần đặt mình vào vị trí cấp dưới, tôn trọng và công bằng với mọi nhân viên.

Bên cạnh đó, các nhà lãnh đạo, quản lý cũng phải quan tâm đến lãnh đạo chuyển đổi. Người lãnh đạo nên trao quyền, động viên và hỗ trợ các nhân viên của mình tham gia vào các hoạt động học tập. Các bộ phận quản lý nhân sự nên chủ động đề xuất các chương trình, hoạt động huấn luyện nhằm nâng cao kỹ năng lãnh đạo trong việc tập trung vào việc xác định mục tiêu, chia sẻ tầm nhìn và truyền cảm hứng cho nhân viên để cùng thực hiện mục tiêu đó. Lãnh đạo cần chia sẻ với nhân viên về định hướng, kế hoạch phát triển của công ty để cho họ thấy được vai trò, tầm quan trọng của công việc hiện nay của họ để họ thấy được sự đóng góp cho công ty và tự hào về công việc của họ. Các doanh nghiệp phải xây dựng văn hóa doanh nghiệp, tạo sự đồng thuận, gắn kết giữa các thành viên trong tổ chức từ cấp lãnh đạo đến nhân viên để cùng vượt qua khó khăn, thách thức nhằm đạt hiệu quả cao trong công việc. Ngoài ra, lãnh đạo cần gia tăng hơn nữa sự đáp ứng nhu cầu về môi trường làm việc, cơ sở vật chất khi làm việc. Môi trường làm việc tốt thì mỗi cá nhân mới có điều kiện làm việc tốt, phát huy khả năng của minh, chung sức thực hiện nhiêm vụ của đơn vị. Đối với thế hệ lao động trẻ hiện nay, đặc biệt là trong lĩnh vực công nghệ thông tin thì bên cạnh chính sách đãi ngộ hợp lý thì họ còn quan tâm đến việc phát triển và khẳng định bản thân tại nơi công tác. Do vậy, lãnh đạo phải thông tin rõ ràng, minh bạch các chế độ đãi ngộ để người lao động gắn kết công việc dài lâu.

Tuy đã đạt được những mục tiêu đề ra ban đầu nhưng nghiên cứu vẫn còn một số hạn chế nhất định và cần được phải tiếp tục bổ sung nghiên cứu như sau: Đầu tiên, nghiên cứu này chưa phân tích và kiểm định tác động của các biến kiểm soát như độ tuổi, giới tính, thâm niên công tác nên trong các nghiên cứu tiếp theo cần phải phân tích, làm rõ vai trò của các yếu tố này. Thứ hai, nghiên cứu sử dụng dữ liệu cắt ngang tại một thời điểm cụ thể do đó không thể suy rộng ra theo thời gian. Hơn nữa, với đối tượng phân tích là các doanh nghiệp công nghệ thông tin với các đặc trưng của nó do vậy không thể đảm bảo tính bền vững của kết quả nghiên cứu khi mở rộng sang các ngành nghề, khu vực khác. Do vậy, cần mở rộng đối tượng khảo sát để hoàn thiện hơn mô hình.

\section{Tài liệu tham khảo}

Akhtar, S., Arif, A., Rubi, E., \& Naveed, S. (2012). Impact of organizational learning on organizational performance: Study of higher education institutes. International Journal of Academic Research, 3(5), 327-331.

Arnold, K. A. (2017). Transformational leadership and employee psychological well-being: A review and directions for future research. Journal of Occupational Health Psychology, 22(3), 381-393.

Baldwin, M. (2016). Social work, critical reflection and the learning organization. London, UK: Routledge.

Bass, B. M. (1985). Leadership and performance beyond expectations. New York, NY: Free Press.

Bass, B. M. (2000). The future of leadership in the learning organization. Journal of Leadership Studies, 7(3), 18-38.

Bass, B. M., \& Riggio, R. E. (2006). Transformational leadership. Mahwah, NJ: Erlbaum. 
Bono, J. E., \& Judge, T. A. (2003). Self-concordance at work: Toward understanding the motivational effects of transformational leaders. Academy of Management Journal, 46(5), 554-571.

Burns, J. M. (1978). Leadership. New York, NY: Free Press.

Campbell, J. P. (1990). Modeling the performance prediction problem in industrial and organizational psychology. In M. D. Dunnette \& L. M. Hough (Eds.), Handbook of industrial and organizational psychology (pp. 687-732). Palo Alto, CA: Consulting Psychologists Press.

Carter, D., \& Baghurst, T. (2014). The influence of servant leadership on restaurant employee engagement. Journal of Business Ethics, 124(3), 453-464.

Chang, S. C., \& Lee, M. S. (2007). A study on relationship among leadership, organizational culture, the operation of learning organization and employees' job satisfaction. The Learning Organization, 14(2), 155-185.

Cho, J., \& Dansereau, F. (2010). Are transformational leaders fair? A multi-level study of transformational leadership, justice perceptions, and organizational citizenship behaviors. Leadership Quarterly, 21(3), 409-421.

Choudhary, A., Akhtar, S. A., \& Zaheer, A. (2013). Impact of transformational and servant leadership on organizational performance: a comparative analysis. Journal of Business Ethics, 116(2), 433-440.

Crippen, C. (2005). Servant-leadership as an effective model for educational leadership and management: First to serve, then to lead. Management in Education, 18(5), 11-16.

Dierendonck, D. V. (2011). Servant leadership: A review and synthesis. Journal of Management, 37(4), 1228-1261.

Dierendonck, D. V., \& Nuijten, I. (2011). The servant leadership survey: Development and validation of a multidimensional measure. Journal of Business and Psychology, 26(3), 249267.

Dierendonck, D. V., Stam, D., Boersma, P., Windt, N. D., \& Alkema, J. (2014). Same difference? Exploring the differential mechanisms linking servant leadership and transformational leadership to follower outcomes. Leadership Quarterly, 25(3), 544-562.

Dimmock, C., \& Walker, A. (2005). Educational leadership: Culture and diversity. Thousand Oaks, CA: Sage.

Ehrhart, M. G. (2004). Leadership and procedural justice climate as antecedents of unit-level organizational citizenship behavior. Personnel Psychology, 57(1), 61-94.

Garcia-Morales, V. J., Lloens-Montes, F. J., \& Verdu-Jover, A. J. (2008). The effects of transformational leadership on organizational performance through knowledge and innovation. British Journal of Management, 19(4), 299-319.

Garcia-Morales, V., Jimenez-Barrionuevo, M. M., \& Gutierrez-Gutierrez, L. J. (2012). Transformational leadership influence on organizational performance through organizational learning and innovation. Journal of Business Research, 65(7), 1040-1050.

Garvin, D., Edmondson, A., \& Gino, F. (2008). Is yours a learning organization? Harvard Business Review, 86(3), 109-134. 
Geier, M. T. (2016). Leadership in extreme contexts: Transformational leadership, performance beyond expectations? Journal of Leadership \& Organizational Studies, 23(3), 234-247.

Gentle, P., \& Clifton, L. (2017). How does leadership development help universities become learning organisations. The Learning Organization, 24(5), 278-285.

Greenleaf, R. K. (1977). Servant leadership. New York, NY: Paulist Press.

Hackman, J. R. (2002). Leading teams: Setting the stage for great performances. Boston, MA: Harvard Business School Press.

Hair, J. F., Hult, G. T. M., Ringle, C., \& Sarstedt, M. (2014). A primer on Partial Least SquaresbStructural Equation Modeling (PLS-SEM). London, UK: Sage Publications.

Hernandez, M., Eberly, M. B., Avolio, B. J., \& Johnson, M. D. (2011). The loci and mechanisms of leadership: Exploring a more comprehensive view of leadership theory. The Leadership Quarterly, 22(6), 1165-1185.

Ho, L. A. (2011). Meditation, learning, organizational innovation and performance. Industrial Management and Data Systems, 111(1), 113-131.

Hoch, J. E., Bommer, W. H., Dulebohn, J. H., \& Wu, D. (2018). Do ethical, authentic, and servant leadership explain variance above and beyond transformational leadership? A meta-analysis. Journal of Management, 44(2), 501-529.

Jacobs, G. A. (2006). Servant leadership and follower commitment. Proceedings of the 2006 Servant Leadership Research Round-table. Retrieved December 14, 2020, from http://www.regent.edu/acad/global/publications/sl_proceedings/2006/gilbert.pdf

Johnson, J. R. (2002). Leading the learning organization: Portrait of four leaders. The Leadership \& Organization Development Journal, 23(5), 241-249.

Jyoti, J., \& Dev, M. (2015). The impact of transformational leadership on employee creativity: The role of learning orientation. Journal of Asia Business Studies, 9(1), 78-98.

Khandekar, A., \& Sharma, A. (2006). Organizational learning and performance: Understanding Indian scenario in present global context. Education and Training, 48(8/9), 682-692.

Lam, Y. J. (2002). Defining the effects of transformational leadership on organisational learning: A cross-cultural comparison. School Leadership \& Management, 22(4), 439-452.

Lanctot, J. D., \& Irving, J. A. (2010). Character and leadership: Situating servant leadership in a proposed virtues framework. International Journal of Leadership Studies, 6(1), 28-50.

Lapointe, É., \& Vandenberghe, C. (2018). Examination of the relationships between servant leadership, organizational commitment, and voice and antisocial behaviors. Journal of Business Ethics, 148(1), 99-115.

Liu, H. (2019). Just the servant: An intersectional critique of servant leadership. Journal of Business Ethics, 156(4), 1099-1112.

López, S. P., Peón, J., \& Ordás, C. (2005). Organizational learning as a determining factor in business performance. The Learning Organization, 12(3), 227-245.

Malcalm, E., \& Tamatey, S. (2017). Examining leadership style on employee performance in the public sector of Ghana. International Journal of Scientific and Research Publications, 7(11), 343-358. 
McClellan, J. L. (2007). The advisor as servant: The theoretical and philosophical relevance of servant leadership to academic advising. NACADA Journal, 27(2), 41-49.

McGill, M. E., \& Slocum, J. W., Jr. (1993). Unlearning the organization. Organizational Dynamics, 22(2), 67-79.

Morgan, R. E., \& Turnell, C. R. (2003). Market-based organizational learning and market performance gains. British Journal of Management, 14(3), 225-274.

Northouse, P. G. (2018). Leadership: Theory and practice. Thousand Oaks, CA: Sage publications.

Olesia, W. S., Namusonge, G., \& Iravo, M. (2014). Servant leadership: The exemplifying behaviours. IOSR Journal of Humanities and Social Science, 19(6), 75-80.

Örtenblad, A. (2018). What does "learning organization" mean? The Learning Organization, 25(3), 150-158.

Parolini, J., Patterson, K., \& Winston, B. (2009). Distinguishing between transformational and servant leadership. The Leadership \& Organization Development Journal, 30(3), 274-291.

Parris, D. L., \& Peachey, J. W. (2013). A systematic literature review of servant leadership theory in organizational contexts. Journal of Business Ethics, 113(3), 377-393.

Peltokorpi, J., \& Niemi, E. (2018). Effects of group size and learning on manual assembly performance: An experimental study. International Journal of Production Research, 57(2), 452-469.

Podsakoff, P. M., MacKenzie, S., \& Bommer, W. H. (1996). Transformational leader behaviors and substitutes for leadership as determinants of employee satisfaction, commitment, trust, and organizational citizenship behaviors. Journal of Management, 22(2), 259-298.

Prugsamatz, R. (2010). Factors that influence organization learning sustainability in non- profit organizations. The Learning Organization, 17(3), 243-267.

Ragnarsson, S., Kristjánsdóttir, E. S., \& Gunnarsdottir, S. (2018). To be accountable while showing care: The lived experience of people in a servant leadership organization. SAGE Open, 8(3), 1-12.

Rhodes, J., Lok, P., Hung, R., \& Fang, S. (2008). An integrative model of organizational learning and social capital on effective knowledge transfer and perceived organizational performance. Journal of Workplace Learning, 20(4), 245-258.

Russell, R. F., \& Stone, A. G. (2002). A review of servant leadership attributes: Developing a practical model. Leadership \& Organization Development Journal, 23(3), 145-157.

Sarros, J. C., Tanewski, G. A., Winter, R. P., Santora, J. C., \& Densten, I. L. (2002). Work alienation and organizational leadership. British Journal of Management, 13(4), 285-304.

Schiena, R. D., Letens, G., Aken, E. V., \& Farris, J. (2013). Relationship between leadership and characteristics of learning organizations in deployed military units: An exploratory study. Administrative Sciences, 3(3), 143-165.

Sendjaya, S. (2015). Personal and organizational excellence through servant leadership: Learning to serve, serving to lead, leading to transform. Cham Heidelberg, NY: Springer.

Sendjaya, S., Sarros, J. C., \& Santora, J. C. (2008). Defining and measuring servant leadership behaviour in organizations. Journal of Management Studies, 45(2), 402-424. 
Senge, P. M. (1990). The fifth discipline: The art and practice of the learning organization. New York, NY: Doubleday.

Singh, S. K. (2008). Relationship between learning organization and transformational leadership: Banking organizations in India. International Journal of Business and Management Science, 1(1), 97-111.

Song, C., Park, K. R., \& Kang, S. W. (2015). Servant leadership and team performance: The mediating role of knowledge-sharing climate. Social Behavior and Personality: International Journal, 43(10), 1749-1760.

Spears, L. C. (2010), Character and servant leadership: Ten characteristics of effective, caring leaders. The Journal of Virtues and Leadership, 1(1), 25-30.

Staats, B. (2015). The adaptable emphasis leadership model: A more full range of leadership. Servant Leadership: Theory \& Practice, 2(2), 12-26.

Stone, G. A., Russell, R. F., \& Patterson, K. (2004). Transformational versus servant leadership: A difference in leader focus. The Leadership \& Organization Development Journal, 25(3/ 4), 349-359.

Tan, S. C., Smyrnios, K. X., \& Xiong, L. (2014). What drives learning orientation in fast growth SMEs? International Journal of Entrepreneurial Behavior and Research, 20(4), 324-350.

Tepper, B. J., Dimotakis, N. E., Lambert, L., Koopman, J., Matta, F. K., Park, H., \& Goo, W. (2017). Examining follower responses to transformational leadership from a dynamic, person-environment fit perspective. Academy of Management Journal, 61(4), 1343-1368.

Tomal, D. R., \& Jones, K. J. (2015). A comparison of core competencies of women and men leaders in the manufacturing industry. The Coastal Business Journal, 14(1), 13-25.

Triana, M. D. C., Richard, O. C., \& Yucel, I. (2017). Status incongruence and supervisor gender as moderators of the transformational leadership to subordinate affective organizational commitment relationship. Personnel Psychology, 70(2), 429-467.

Veelen, R. V., \& Ufkes, E. G. (2017). Teaming up or down? A multisource study on the role of team identification and learning in the team diversity-performance link. Group \& Organization Management, 44(1), 38-71.

Voon, M. L., Lo, M. C., Ngui, K. S., \& Ayob, N. B. (2011). The influence of leadership styles on employees' job satisfaction in public sector organizations in Malaysia. International Journal of Business, Management and Social Sciences, 2(1), 24-32.

Xie, L. (2019). Leadership and organizational learning culture: A systematic literature review. European Journal of Training and Development, 43(1/2), 76-104.

Xie, L. (2020). The impact of servant leadership and transformational leadership on learning organization: A comparative analysis. Leadership \& Organization Development Journal, 41(2), 220-236.

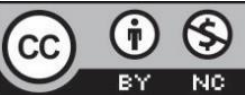

Creative Commons Attribution-NonCommercial 4.0 International License. 